\title{
Expérience acquise en France sur la radiotoxicologie des composés industriels de l'uranium
}

\author{
J. CHALABREYSSE*, P. BEAU**, C. CHEVALIER ${ }^{\star \star \star}$, L. JEANMAIRE* \\ G. BATALLER ${ }^{\star \star \star \star}$, P. BERARD*, B. GIBERT ${ }^{\star \star \star \star \star}$
}

(Manuscrit reçu le 19 septembre 1988)

\begin{abstract}
RÉSUMÉ
Les auteurs, membres de différentes organisations et sociétés industrielles, présentent leurs observations menées depuis une trentaine d'années en France, en surveillance systématique ou après incidents de contamination par des composés d'uranium. A partir de leurs résultats, ils proposent des valeurs de référence et des recommandations pour la surveillance radiotoxicologique des travailleurs exposés aux composés industriels d'uranium.
\end{abstract}

\section{ABSTRACT}

The authors, representing several organisations and industrial firms, present observations conducted for some thirty years in France, including routine monitoring or special measurements following contamination by uranium compounds. In analysing the results, they propose reference values and recommendations for radiotoxicological monitoring of workers exposed to industrial uranium compounds.

- Commissariat à l'énergie atomique (CEA), Institut de protection et de sûreté nucléaire, Département de protection sanitaire, Service d'hygiène industrielle, CEN-VALRHO, BP 38, 86700 Pierrelatte.

** Commissariat à l'énergie atomique (CEA), Institut de protection et de sûreté nucléaire. Département de protection sanitaire, BP 6, 92265 Fontenay-aux-Roses Cedex.

*** EDF, Laboratoire d'analyses médicales et radiotoxicologiques, BP 114, 93203 St-Denis Cedex.

**** Commissariat à l'énergie atomique (CEA), Centre d'études nucléaires de Cadarache, Laboratoire d'analyses médicales, 13108 St-Paul-lez-Durance Cedex 147.

***** Société Comurhex, Malvési, BP 222, 11102 Narbonne Cedex. 


\section{INTRODUCTION}

L'expérience, en France, sur les risques réels liés à la mise en œuvre industrielle de composés de l'uranium remonte aux années 50 avec le Centre du Bouchet. En 1962, a démarré la construction de l'usine militaire d'enrichissement de Pierrelatte à laquelle était associée une usine chimique de production d'UF $F_{6}$. A présent, le cycle de l'uranium est complètement réalisé en France.

A l'initiative du conseiller médical du CEA, un groupe de travail a été créé en 1975, comportant des spécialistes français (CEA, Cogema, EDF, Comurhex, FBFC, $\mathrm{SICN}$ ) de la radiotoxicologie de l'uranium. Ce groupe de travail s'appuie sur le Service d'hygiène industrielle qui, d'une part, assure, en liaison avec les différents services médicaux du travail, la surveillance opérationnelle de la plupart des établissements industriels en fonctionnement normal et lors d'incidents, et d'autre part, pour compléter les connaissances sur le métabolisme et la toxicité, conduit des études expérimentales, dans le cadre d'un contrat avec la Commission des communautés européennes (1). Ainsi, la radiotoxicologie de l'uranium fait l'objet en France d'un programme complet:

- surveillance opérationnelle des établissements industriels en fonctionnement normal ou lors d'incidents,

- études expérimentales sur la toxicité et le métabolisme,

- élaboration d'une doctrine et de valeurs de référence pour permettre l'exploitation industrielle en conformité avec la réglementation.

\section{OBSERVATIONS EXEMPLAIRES EN SITUATIONS INDUSTRIELLES}

\subsection{Conditions réelles d'exposition}

La fabrication du combustible à l'oxyde d'uranium nécessite l'élaboration d'un certain nombre de composés chimiques. Leur technologie de production, n'étant pas toujours réalisée en enceinte de confinement, il n'y a pas de barrière absolue entre ces composés et le personnel. II en résulte que, dans les conditions normales de fonctionnement, se produit une contamination atmosphérique a minima responsable d'une exposition interne chronique et de bas niveau du personnel, majorée lorsque le fonctionnement des installations est perturbé. Pour les autres composés, leur fabrication et leur utilisation se font dans des conditions de confinement telles que seule une exposition consécutive à leur libération accidentelle est à envisager.

(1) Programme Radioprotection, Contrat B 160088-F-CD. 
Les composés de l'uranium auxquels sont habituellement exposés les ouvriers sont principalement des corps sous forme pulvérulente: uranates d'ammonium et de magnésium, trioxyde et tétrafluorure d'uranium, dioxyde et sesquioxyde d'uranium. Les conditions de production de ces composés (température, acidité ou alcalinité, etc.) étant très différentes, leur granulométrie est très variable. Elle s'écarte souvent de façon notable de la granulométrie de référence (DAMA de $1 \mu \mathrm{m}$ adopté par la Commission internationale de protection radiologique - CIPR) pour prendre des valeurs plus élevées. L'hexafluorure d'uranium et son produit de décomposition, l'oxyfluorure, qui n'apparaissent que dans des conditions accidentelles, se présentent à l'état de vapeur, pour le premier, et, pour le second, de particules fines qui peuvent s'agglomérer pour donner des agrégats dont la taille peut s'élever jusqu'à $10 \mu \mathrm{m}$.

Les procédés de fabrication assurent aux produits une pureté qui peut être altérée lorsque les paramètres de réaction n'ont plus leur valeur nominale. II en résulte la production de mélanges: par exemple, $U_{4}+U_{2} \mathrm{O}_{2} F_{2}$ (le pourcentage d'UO $\mathrm{F}_{2} \mathrm{~F}_{2}$ est normalement très faible). Les dérèglements de fonctionnement sont souvent générateurs de contamination atmosphérique et, par conséquent, l'inhalation de mélanges par les ouvriers est une éventualité à considérer.

\subsection{Exposition à des composés de la classe D}

Nous mentionnerons les résultats observés à la suite d'un incident (libération d'UF ${ }_{6}$ ), survenu en mai 1965 dans l'usine pilote de Pierrelatte, au cours duquel il fut possible de procéder à un très grand nombre de contrôles sur un très grand nombre d'individus: les urines constituent la voie majeure de l'excrétion, très importante dans les premières heures qui suivent l'absorption.

\subsection{Exposition à des composés de la classe $W$}

Nous disposons de résultats accumulés depuis 1959 pour un établissement où l'on produit du tétrafluorure d'uranium à partir des différents composés uranifères livrés. Une partie du tétrafluorure est ensuite transformée en uranium métal, par magnésiothermie, l'autre est expédiée dans une autre usine pour fabrication de l'hexafluorure.

L'exposition des travailleurs aux poussières uranifères est le risque majeur. Elle rend nécessaire une surveillance permanente et systématique des conditions de travail et des travailleurs eux-mêmes; elle s'exerce, en moyenne, sur 200 salariés par an, depuis 20 ans. De 1960 à 1972, les taux d'excrétion urinaire ont oscillé entre 10 et $40 \mu \mathrm{g}$ par litre, avec parfois des pics isolés à 100 et même $200 \mu \mathrm{g}$ qui revenaient en quelques jours aux valeurs habituellement rencontrées. Le retour à ces valeurs était beaucoup plus rapide que celui auquel on aurait pu s'attendre. Depuis 1979, l'augmentation du rythme de production a imposé une méthode de surveillance plus stricte et mieux codifiée. 
De cette expérience industrielle, on peut tirer trois observations:

- la corrélation entre les résultats urinaires et les prélèvements atmosphériques au poste de travail n'est pas réalisable directement car soit les travailleurs portent un masque, soit ils se déplacent dans plusieurs ateliers;

- le mode d'incorporation est chronique, révélé par un fond continu de contamination sur lequel émerge des pics ayant pour origine des dysfonctionnements du procédé industriel avec absence du port de la protection individuelle (masque);

- ces pics d'excrétion urinaire reviennent rapidement au niveau du fond continu de contamination chronique; néanmoins, subsiste la signification du niveau de base des pics d'excrétion rapide; est-ce dû aux impuretés amenées par le procédé ou bien, hypothèse la plus probable, est-ce la mise en évidence d'une plus grande transférabilité de ces composés que celle de la classe W?

\subsection{Exposition à des composés de la classe $Y$}

L'expérience est plus récente. Pour l'illustrer, nous mentionnerons, deux cas:

- Exposition à un mélange $\mathrm{UO}_{2}-U_{3} \mathrm{O}_{8}$

L'agent travaillait au poste de pastillage d'oxyde d'uranium, pour lequel la surveillance repose sur deux anthropogammamétries, deux urines et deux selles par an. A un examen systématique, la charge pulmonaire a dépassé le niveau d'exclusion. L'exclusion du poste a été immédiate et les contrôles ont été effectués tous les mois. A partir de son exclusion, l'agent a présenté une excrétion urinaire en uranium plus importante que celle observée pendant l'exposition; l'évolution de la rétention pulmonaire a mis en évidence une période biologique d'environ 140 jours. Le rapport entre l'excrétion fécale et la rétention pulmonaire est voisin de 1 pour 1000 .

\section{- Exposition à $\mathrm{UO}_{2}$}

Après exclusion du poste de travail, nous avons pu observer que la rétention pulmonaire était conforme à celle fixée par le modèle pulmonaire de la CIPR (période biologique apparente d'environ $490 \mathrm{j}$ ), le rapport entre excrétion fécale et rétention pulmonaire est voisin de 1 pour 1000 (excepté les premiers jours). Par contre, l'excrétion urinaire est supérieure à celle prévue dans le modèle de la CIPR. 


\section{DISCUSSION}

Vingt-cinq ans d'expérience de la surveillance de l'industrie de l'uranium conduisent aux conclusions et propositions suivantes:

\subsection{Transférabilitè et toxicité réelles des composés industriels français}

\section{- Composés D:}

leur comportement est globalement conforme à celui retenu par la CIPR;

la toxicité chimique rénale est moins importante que celle signalée dans la littérature, nous n'avons jamais observé de troubles rénaux à court terme, même après des incidents ayant entraîné des concentrations urinaires supérieures à $1,5 \mathrm{mg} / \mathrm{l}$ d'urine.

\section{- Composés W:}

l'UF $_{4}$ n'a pas un comportement parfaitement tranché, ce qui peut être dû, dans un certain nombre de cas, à la présence d' $\mathrm{UO}_{2} \mathrm{~F}_{2}$ au titre d'impuretés industrielles;

des excrétions chroniques, durant de longues périodes, allant jusqu'à $40 \mu \mathrm{g}$, avec des pics isolés à 100 et même $200 \mu \mathrm{g} / \mathrm{l}$ d'urine n'ont pas provoqué d'atteintes biologiques.

\section{- Composés Y:}

Selon les composés, nous observons 2 types de période biologique au niveau pulmonaire $\left(\mathrm{U}_{3} \mathrm{O}_{8}: 140 \mathrm{j} ; \mathrm{UO}_{2}: 490 \mathrm{j}\right)$. Ceci demande à être approfondi et vérifié.

\subsection{Valeurs de référence}

Les situations industrielles sont bien différentes, par leur complexité et leurs aléas, des situations simples et clairement définies dans des schémas théoriques. II est indispensable de concilier nécessités industrielles et impératifs de la protection.

En marche industrielle, les résultats radiotoxicologiques doivent être replacés dans leur contexte; un résultat isolé a peu de signification; il faut observer l'évolution dans le temps des résultats individuels et des groupes d'individus affectés au même poste de travail.

Néanmoins, pour éviter tout excès préjudiciable à la protection, nous proposons des valeurs destinées à servir de borne de référence pour l'appréciation du risque réel. Ces valeurs tiennent compte simultanément du mode de contamination (unique, connue ou inaperçue, et chronique), des périodicités réalistes de surveillance individuelle, de la sensibilité des méthodes de mesures, de la toxicité chimique et de la toxicité radiologique de l'uranium, suivant la nature du composé et la teneur en isotopes 235 et 234. 


\subsubsection{Limites d'incorporation}

Cette évolution de la toxicité (chimique, radiologique) de l'uranium se traduit dans les valeurs des limites d'incorporation (tableau I).

\section{TABLEAU ।}

\section{Limites d'incorporation suivant le type de composés}

\begin{tabular}{|c|c|c|}
\hline Limite d'incorporation & $\begin{array}{c}\text { Radiotoxicité } \\
\text { (LA) } \\
\text { en Bq } \\
(\mathrm{a})\end{array}$ & $\begin{array}{c}\text { Chimiotoxicité } \\
\text { en } \mu \mathrm{g} \\
(\mathrm{b})\end{array}$ \\
\hline Type de composés & $5.10^{4}$ & $2,5.10^{3}$ \\
\hline W & $2,8.10^{4}$ & $11.10^{3}$ \\
\hline Y & $1,5.10^{3}$ & $210.10^{3}$ \\
\hline
\end{tabular}

LAI: Limite annuelle d'incorporation.

On peut calculer l'activité spécifique du mélange d'isotopes d'uranium correspondant à la transition chimiotoxicité-radiotoxicité (tableau II).

TABLEAU ॥

Activité spécifique du mélange, exprimée en Bq. $\mu \mathbf{g}^{-1}$, correspondant à la transition chimiotoxicité-radiotoxicité

\begin{tabular}{|c|c|c|c|}
\hline Incorporation & Classe D & Classe W & Classe Y \\
\hline $\begin{array}{c}\text { Chronique } \\
\frac{\mathrm{a}}{250} \times \frac{1}{\mathrm{~b}}\end{array}$ & $\begin{array}{c}\text { Chimiotoxique } \\
<0,08< \\
\text { Radiotoxique }\end{array}$ & $\begin{array}{c}\text { Radiotoxique } \\
\left(1.10^{-2}\right)\end{array}$ & $\begin{array}{c}\text { Radiotoxique } \\
\left(3.10^{-5}\right)\end{array}$ \\
\hline Unique & Chimiotoxique & $\begin{array}{c}\text { Chimiotoxique } \\
<0,6< \\
\text { Radiotoxique }\end{array}$ & $\begin{array}{c}\text { Radiotoxique } \\
\left(2.10^{-3}\right)\end{array}$ \\
\hline
\end{tabular}

\section{Remarques}

250 représente le nombre de jours réels durant lesquels s'effectue l'exposition chronique; a et b sont tirés du tableau I: 4 représente le nombre de contrôles par an.

Les valeurs d'activité spécifique inscrites dans ce tableau rendent compte de la transition toxicité chimique $\rightarrow$ toxicité radiologique.

Pour les compossés de la classe D, la radiotoxicité devient prépondèrante en exposition chronique à partir de $3 \%$ en 235 .

Pour les composés de la classe $\mathrm{W}$, la radiotoxicité devient prépondérante en exposition unique à partir de $30 \%$ en ${ }^{235} \mathrm{U}$. 


\subsubsection{Valeurs radiotoxicologiques de référence en surveillance industrielle}

- Pour tenir compte de la double contrainte des deux types de toxicité de l'uranium (chimique et radiologique) liés, notamment, à la variation de l'activité spécifique des mélanges isotopiques en fonction de l'enrichissement en ${ }^{235} \mathrm{U}$, on exprime les valeurs de référence en unités pondérales (microgrammes) et/ou unité d'activité alpha (becquerels), une des deux expressions représentant le facteur limitant suivant le cas considéré.

- En ce qui concerne la toxicité chimique, on distinguera, au niveau rénal, deux situations:

- l'une correspondant à une excrétion urinaire quotidienne d'uranium (contamination chronique);

- l'autre correspondant à une excrétion urinaire faisant suite à une absorption élevée, mais unique d'uranium (incident).

Pour la première situation, on a retenu $70 \mu \mathrm{g}$ pour les urines de $24 \mathrm{~h}$ (soit environ $50 \mu \mathrm{g} / \mathrm{l}$ d'urine, ou $35 \mu \mathrm{g} / \mathrm{g}$ de créatinine). II s'agit d'une valeur empirique que l'on peut assimiler à un niveau d'investigation dérivé.

Pour la deuxième situation, la valeur urinaire est dérivée de la limite maximale à l'inhalation en une fois c'est-à-dire $2,5 \mathrm{mg}$. On obtient $490 \mu \mathrm{g}$ dans les urines des premières $24 \mathrm{~h}$, ce qui constitue ainsi la limite maximale urinaire quel que soit le type de composés ( $D$ ou $W$ ); cette valeur permet de remonter à la limite d'incorporation $\left(11 \times 10^{3} \mu \mathrm{g}\right)$ pour les composés $\mathrm{W}$ en considérant la chimiotoxicité (tableau I).

- En ce qui concerne la toxicité radiologique, on considère qu'il est réaliste et raisonnable de pratiquer en milieu industriel un contrôle individuel une fois par trimestre. Sur ce mode de surveillance, on détermine des valeurs d'alerte qui, si elles sont atteintes, révèlent que les conditions d'exposition en cours de fonctionnement, hors incident, se dégradent et nécessitent, d'une part, une étude des postes de travail pour éviter la poursuite de telles expositions et, d'autre part, des examens complémentaires sur l'homme pouvant rendre nécessaire une exclusion de toute contamination pendant la durée des investigations. Le calcul est basé sur l'incorporation chaque jour, durant $90 \mathrm{j}$, de $\mathrm{LAl} / 250$ (90 j résultant d'une périodicité de 4 contrôles par an; 250 représentant le nombre de jours de travail effectif durant 1 an). On détermine pour chaque type d'examen (urine, selles, poumon) le niveau d'investigation dérivé qui correspond au 3/10 de l'incorporation chronique de la LAl/250.

- En fonctionnement industriel réel, deux autres situations peuvent être rencontrées:

- au cours de la surveillance systématique, basée sur un rythme trimestriel, on détecte une contamination individuelle alors qu'il n'existe pas de contamination chronique révélée par les contrôles d'ambiance systématiques;

- un dysfonctionnement manifeste du procédé industriel s'est produit pouvant entraîner une contamination des travailleurs présents. 
Pour ces deux situations, on retient comme base du calcul la valeur de $\mathrm{LAl} / 4$. On détermine les niveaux de contamination pour les 3 types d'examens radiotoxicologiques (urines, selles, poumon), en supposant, pour la première situation, qu'il s'est produit une contamination unique, qui est passée inaperçue, au milieu (45 j) de l'intervalle de contrôle, et, pour la deuxième situation, on effectue les calculs de l'activité à laquelle on peut s'attendre les premières $24 \mathrm{~h}$ après le dysfonctionnement du procédé (tableau III).

\section{TABLEAU III}

Valeurs de référence en surveillance radiotoxicologique industrielle

\begin{tabular}{|c|c|c|c|c|c|c|}
\hline \multirow{3}{*}{ Composé } & \multirow{2}{*}{\multicolumn{2}{|c|}{$\begin{array}{c}\text { Mode de } \\
\text { contamination }\end{array}$}} & \multirow{3}{*}{$\begin{array}{c}\text { Délai } \\
\text { d'examen } \\
\text { (en jours) }\end{array}$} & \multicolumn{3}{|c|}{ Valeurs de référence } \\
\hline & & & & \multirow{2}{*}{$\begin{array}{c}\text { Urines } \\
(24 \mathrm{~h}) \\
(\mathrm{LD}=6 \mu \mathrm{g} \\
\text { ou } \\
\left.1,5.10^{-2} \mathrm{~Bq}\right)\end{array}$} & \multirow{2}{*}{$\begin{array}{c}\text { Selles } 24 \mathrm{~h} \\
(\mathrm{LD}=0,1 \mu \mathrm{g} \\
\text { ou } \\
\left.4.10^{-3} \mathrm{~Bq}\right)\end{array}$} & \multirow[b]{2}{*}{$\begin{array}{c}\text { Poumon } \\
(L D= \\
150 \mathrm{~Bq})\end{array}$} \\
\hline & Type & Circonstances & & & & \\
\hline \multirow{3}{*}{ D } & \multirow[t]{2}{*}{ Unique } & Connu & 1 & $490 \mu \mathrm{g}$ & $100 \mu \mathrm{g}$ & $<$ LD \\
\hline & & Inaperçu & 45 & $\begin{array}{c}2 \mu \mathrm{g} \mathrm{et} / \mathrm{ou} \\
6 \mathrm{~Bq}\end{array}$ & $<$ LD & $<\mathrm{LD}$ \\
\hline & Chronique & Connu & 90 & $\begin{array}{c}70 \mu \mathrm{g} \mathrm{et} / \mathrm{ou} \\
29 \mathrm{~Bq}\end{array}$ & $\begin{array}{c}26 \mu \mathrm{g} \mathrm{et} / \mathrm{ou} \\
10 \mathrm{~Bq}\end{array}$ & $<$ LD \\
\hline \multirow{3}{*}{ W } & \multirow[t]{2}{*}{ Unique } & Connu & 1 & $\begin{array}{c}490 \mu \mathrm{g} \\
\text { et/ou } \\
300 \mathrm{~Bq}\end{array}$ & $\begin{array}{c}440 \mu \mathrm{g} \\
\text { et/ou } \\
300 \mathrm{~Bq}\end{array}$ & $\begin{array}{c}<\mathrm{LD} \\
\mathrm{et} / \mathrm{ou} \\
1400 \mathrm{~Bq}\end{array}$ \\
\hline & & Inaperçu & 45 & $\begin{array}{c}6 \mu \mathrm{g} \mathrm{et} / \mathrm{ou} \\
3,5 \mathrm{~Bq}\end{array}$ & $\begin{array}{l}8 \mu \mathrm{g} \\
\text { ettou } \\
5 \mathrm{~Bq}\end{array}$ & $\begin{array}{c}<\mathrm{LD} \\
\text { et/ou } \\
600 \mathrm{~Bq}\end{array}$ \\
\hline & Chronique & Connu & 90 & $4 \mathrm{~Bq}$ & $18 \mathrm{~Bq}$ & $275 \mathrm{~Bq}$ \\
\hline \multirow{3}{*}{ Y } & \multirow[t]{2}{*}{ Unique } & Connu & 1 & $1 \mathrm{~Bq}$ & $18 \mathrm{~Bq}$ & $<$ LD \\
\hline & & Inaperçu & 45 & $1.10^{-2} \mathrm{~Bq}$ & $5.10^{-2} \mathrm{~Bq}$ & $<$ LD \\
\hline & Chronique & Connu & 90 & $\begin{array}{c}5.10^{-2} \\
\mathrm{~Bq}\end{array}$ & $1 \mathrm{~Bq}$ & $<\mathrm{LD}$ \\
\hline
\end{tabular}

LD = Limite de détection. Délai d'examen : nombre de jours entre la date de contamination (connue ou supposée) et le contrôle radiotoxicologique.

Pour les composés W, au niveau du poumon, les valeurs de référence sont <LD pour les teneurs en $235 \mathrm{U}$ $<30 \%$; les valeurs de $1400 \mathrm{~Bq}$ et $600 \mathrm{~Bq}$ s'appliquent pour des teneurs en ${ }^{235} \mathrm{U}>30 \%$. 


\subsection{Modalités pratiques de surveillance en situation industrielle}

Notre expérience nous a montré que la rétention et l'excrétion sont fortement influencées par trois paramètres: la transférabilité (solubilité biologique), la taille des particules, le rythme de contamination (aigu - chronique).

II en résulte les conséquences suivantes:

1. II est illusoire de vouloir établir un modèle unique de surveillance et, pour chaque installation, une étude basée sur les connaissances théoriques doit être complétée par la prise en compte des possibilités pratiques de détection, des données physico-chimiques et biologiques acquises.

2. La définition de l'agent contaminant est indispensable. En l'absence de données réelles, la transférabilité sera définie par le tableau IV et la granulométrie de l'aérosol sera supposée égale à $1 \mu \mathrm{m}$.

TABLEAU $V$

Classement des composés de l'uranium en fonction de leur transférabilité

\begin{tabular}{|c|c|c|}
\hline $\begin{array}{c}\text { Fortement } \\
\text { transférable }(\mathrm{D}) \\
\text { période }: 0,5 \mathrm{j}\end{array}$ & $\begin{array}{c}\text { Moyennement } \\
\text { transférable }(\mathrm{W}) \\
\text { période }: 50 \mathrm{j}\end{array}$ & $\begin{array}{c}\text { Faiblement } \\
\text { transférable }(\mathrm{Y}) \\
\text { période }: 500 \mathrm{j}\end{array}$ \\
\hline $\mathrm{UF}_{6}$ & $\mathrm{UO}_{3}$ & $\mathrm{UO}_{2}$ \\
$\mathrm{UO}_{2} \mathrm{~F}_{2}$ & $\mathrm{UF}_{4}{ }^{*}$ & $\mathrm{U}_{3} \mathrm{O}_{8}$ \\
$\mathrm{UO}_{2}\left(\mathrm{NO}_{3}\right)_{2}$ & $\mathrm{UCl}_{4}$ & \\
$\mathrm{U}_{2} \mathrm{O}_{7}\left(\mathrm{NH}_{4}\right)_{2}{ }^{*}$ & & hydrures d'uranium \\
sulfates d'uranium & & carbures d'uranium \\
carbonates d'uranium & & \\
\hline
\end{tabular}

- Appartenance non définitivement tranchèe

3. Le mode de contamination peut être unique ou chronique sans que l'on puisse déterminer exactement la part de chacun. II est donc souhaitable que le contrôle de routine puisse assurer raisonnablement ces deux possibilitès.

4. Le choix des analyses à effectuer et de leur rythme dans le cadre d'une surveillance systematique est établi en tenant compte des indications suivantes:

a) Pour les espèces très transférables (classe D), il n'y a pas d'accumulation au niveau pulmonaire et l'excrétion urinaire, plus que fécale, représente le moyen essentiel de surveillance, étant donné que le risque toxique non radiologique se situe au niveau rénal. Deux situations se présentent selon le composé concerné:

- s'il s'agit d'un composé hautement confiné $\left(U_{6}\right)$ dont la libération ne peut être qu'accidentelle, le recueil de l'urine des premières $24 \mathrm{~h}$ est primordial, car il permet d'évaluer l'importance de l'agression rénale. Le suivi de 
l'excrétion des jours suivants est également important par l'indication qu'il donne du niveau de la charge de l'organe et de son élimination. Les valeurs limites de référence proposées doivent être utilisées dans l'esprit de la toxicologie chimique axée sur la notion de seuil. Elles doivent être associées à l'emploi d'indicateurs biologiques permettant de préciser le comportement individuel par rapport au seuil théorique (glucose, gamma GT urinaires).

- Dans le cas d'un composé peu confiné et donnant lieu à des contaminations repétées et de bas niveau, l'excrétion urinaire ne représente que l'incorporation du jour et de la veille. Un contrôle pendant les jours ouvrables reflète donc l'exposition des travailleurs; un contrôle après un repos d'une dizaine de jours donne des indications sur la contamination au niveau du squelette et du rein, reflétant alors l'excrétion plus lente des tissus de stockage. Les valeurs de l'excrétion urinaire que l'on peut obtenir par calcul à partir des modèles se réfèrent à une situation théorique qui n'a pas de réalité industrielle. Ces valeurs élevées sont difficilement admissibles si on les compare aux valeurs retenues pour l'excrétion d'autres métaux lourds (plomb, mercure). Une valeur de $70 \mu \mathrm{g} / \mathrm{j}\left(50 \mu \mathrm{g} . \mathrm{I}^{-1}\right)$ a été adoptée et respectée depuis des années par l'industrie de l'uranium; elle n'a, à long terme, entraîné l'apparition d'aucun trouble rénal. Elle semble donc devoir être conservée. Etant donné la rapidité d'élimination des composés, le contrôle doit être fait de façon assez rapprochée et par roulement plutôt que par séries. L'étalement dans le temps évite d'ignorer les contaminations accidentelles.

b) Pour les composés moyennement transférables (classe W) les valeurs proposées montrent que le contrôle peut être obtenu sans difficulté par des mesures sur les urines et les selles. Le contrôle de la charge pulmonaire est plus difficile, mais au niveau de la LAI, il peut s'effectuer sans problème.

c) Pour les composés faiblement transférables (classe Y), l'excrétion urinaire est négligeable, l'excrétion fécale très faible et la détection du NID au niveau thoracique est hors de portée des installations de mesure. Dans ce type de contamination, l'organe cible de l'irradiation étant le poumon, le contrôle sera basé essentiellement sur la surveillance in vivo d'un niveau maximal admissible pulmonaire. Ce niveau de l'ordre de $740 \mathrm{~Bq}$ correspond à la quantité d'uranium qui, présente en permanence, délivre une irradiation pulmonaire continue bien définie et tolérable. Elle ne préjuge pas de la solubilité des composés; elle est valable aussi bien pour les composés $\mathrm{Y}$ et $\mathrm{W}$. Dans le cas des composés $\mathrm{Y}$, cette valeur est atteinte à $10^{4}$ jours. Une contamination chronique plus importante permettrait d'atteindre plus rapidement ce niveau tolérable (de même, une contamination unique importante pourrait avoir le même résultat), mais alors toute contamination supplémentaire devrait être exclue sous peine de dépasser le niveau, car, théoriquement, le contaminant reste fixé dans le poumon.

II faut donc fixer un niveau d'activité qui sera un niveau d'action (enquête, modification des conditions de travail, exclusion, etc.), à une valeur acceptable dans son principe et réaliste parce que mesurable. Ce niveau devrait se situer à $150 \mathrm{~Bq}$, limite de détection au niveau pulmonaire. 
Si l'on a affaire à des composés de type $\mathrm{W}$, ce que les mesures complémentaires permettront de révéler, on est bien en dessous du NID; par contre, si on a affaire à des composés de type $Y$, le NID est dépassé. En pratique, il ne semble pas que la réalité corresponde bien avec la théorie, car, dans les cas de contamination avec les produits réputés peu transférables, on constate fréquemment que la contamination pulmonaire diminue avec le temps et que certaines urines contiennent de l'uranium.

Le contrôle fondamental doit être réalisé in vivo, ce qui n'empêche pas de le compléter par des analyses d'urine et de selles qui ne devraient avoir qu'un rôle indicatif. La valeur d'excrétion pulmonaire par voie fécale pourrait être fixée à 1 pour 1000 .

5. Le contrôle ne doit pas être seulement le contrôle médical de l'individu, mais également celui de l'environnement. Le progrès de la surveillance passe, en particulier, par la connaissance du milieu de travail, la détermination des propriétés physico-chimiques réelles du contaminant (transférabilité, granulométrie). Le remplacement de données théoriques par des valeurs réelles permet de mieux définir la protection, et l'expérience d'auteurs étrangers montre que ceci s'obtient au prix de contraintes plutôt moins strictes.

\section{CONCLUSIONS}

L'expérience en France de la surveillance radiotoxicologique réelle, en milieu industriel, permet de bien maîtriser un certain nombre de points fondamentaux en conformité avec les recommandations de la CIPR. II s'avère, néanmoins, que certaines observations qui ont pu être faites révèlent des comportements biologiques qui s'écartent du modèle théorique, ce qui pourrait conduire à une révision des paramètres de transférabilité dans le sens d'une élévation de celle-ci, donc des contraintes moins importantes au niveau des normes de surveillance individuelle. En France, il se poursuit un certain nombre de travaux portant sur la solubilité in vitro couplés aux expérimentations animales sur les effets biotoxicologiques de l'uranium, métal lourd, et sur le comportement biologique des composés de type $\mathrm{UO}_{2}$.

\section{RÉFÉRENCES}

[1] BEAU P.G., CAMARASA J. - Intérêt de l'association du retour d'expérience à une modélisation sur des données de la CIPR pour une meilleure évaluation de l'exposition interne: cas des composés de l'uranium naturel. In: Colloque franco-italien sur les expositions professionnelles, SFRPIAIRP, Nice Acropolis, 29-31 janvier 1986. Fontenay-aux-Roses: SFRP, 1986, 287-304.

[2] CAMARASA J., CHALABREYSSE J. - Contribution à la surveillance du personnel exposé à des composés non transférables de l'uranium. Radioprotection, 1980, 15 (1) 3-18. 
[3] CHALABREYSSE J. - Surveillance radiotoxicologique du personnel de l'usine de séparation des isotopes de l'uranium par diffusion gazeuse de Pierrelatte. Méthodes et résultats. Rapport COGEMA-R-2, 1978.

[4] GROUPE DE TRAVAIL URANIUM (GT 8/1). - Valeurs de référence et leur application pour la surveillance de l'exposition professionnelle aux composés de l'uranium naturel. Rapport CEA-R-5370, 1986.

[5] MICHAUD F., ARCHIMBAUD M., CHALABREYSSE J. - Evaluation de la teneur en uranium naturel des urines et des selles chez des sujets non exposés. Radioprotection, 1985, 20 (4) 345-346.

[6] PIECHOWSKI J., MENOUX B. - Rétention et excrétion des radionucléides après incorporation par inhalation chez l'homme adulte. Rapport CEA-R-5266, 1984. 